\title{
La Pteridoflora del Parque Nacional Los Mármoles, Hidalgo, México
}

\author{
Said Ramírez-Cruz ${ }^{1,3}$, Arturo Sánchez-González y y Daniel Tejero-Díez ${ }^{2}$ \\ ${ }^{1}$ Centro de Investigaciones Biológicas, Universidad Autónoma del Estado de Hidalgo (UAEH), \\ Ciudad Universitaria; Carretera Pachuca-Tulancingo km. 4.5, Mineral de la Reforma, Hidalgo, 42184. \\ ${ }^{2}$ Facultad de Estudios Superiores Iztacala, Universidad Nacional Autónoma de México. \\ Carrera de Biología. Apartado Postal 314. Tlalnepantla, Estado de México. 54090. \\ ${ }^{3}$ Autor para la correspondencia. Correo-e: arturosg@uaeh.edu.mx
}

\begin{abstract}
Resumen: El conocimiento sobre las especies de licopodios y helechos en el estado de Hidalgo es escaso, el objetivo principal de esta investigación fue contribuir al conocimiento de la riqueza y distribución de la pteridoflora en diferentes comunidades vegetales del parque nacional Los Mármoles (PNM), la segunda área natural protegida con mayor extensión territorial del estado de Hidalgo. El estudio se basó en la recolección exhaustiva de ejemplares en distintos tipos de vegetación. Se encontraron 11 familias, 28 géneros y 65 especies de helechos y una familia con seis especies de licopodios. Se citan siete nuevos registros para el estado de Hidalgo: Anemia mexicana var. makrinii, Argyrochosma palmeri, Cheilanthes spiculata, Pecluma alfredii var. cupreolepis, Polypodium puberulum, Selaginella sartorii y $S$. wrightii.
\end{abstract}

Palabras clave: licopodios, helechos, área natural protegida, análisis de agrupamiento

\begin{abstract}
The objective of the present study was to document the richness and distribution of the pteridoflora in the plant communities of the National Park Los Mármoles, the second largest natural protected area and the largest in Hidalgo. The study was based on exhaustive collection of specimens in different types of vegetation. Eleven families, 28 genera and 65 species of ferns were found, and one family with six species of lycopods. Seven taxa not previously mentioned for the state of Hidalgo are cited: Anemia mexicana var. makrinii, Argyrochosma palmeri, Cheilanthes spiculata, Pecluma alfredii var. cupreolepis, Polypodium puberulum, Selaginella sartorii and S. wrightii.
\end{abstract}

Key words: Ferns, lycophytes, natural protected areas, cluster analysis

$\mathbf{L}^{2}$ a pteridoflora mexicana está compuesta por aproximadamente 1,008 especies y 16 variedades o subespecies (Mickel y Smith, 2004), lo cual representa el $4.3 \%$ de la flora vascular de México, calculada en 23,359 especies (Villaseñor, 2003). Entre las diferentes regiones del país, el estado de Hidalgo, tiene una posición geográfica privilegiada y una heterogeneidad fisiográfica marcada, por lo que debería tener una elevada riqueza florística; en el caso de los helechos y licopodios, quizá debería estar en quinto lugar, después de Oaxaca, Chiapas, Veracruz y Puebla. Sin embargo, Mickel y Smith (2004) citan la presencia de 258 especies, número semejante o inferior al de estados como Guerrero (373; Lorea-Hernández y Velázquez Montes, 1998), Estado de México (257; Tejero-Díez, 2007), More- los (158; Riba, et al., 1996) o la región de Nueva Galicia (281; Mickel, 1992), que son más secos en promedio. Ello se debe a la ausencia de estudios locales y a que los pocos existentes no son exhaustivos en este rubro. En diversos estudios florísticos llevados a cabo en el estado de Hidalgo (Luna-Vega et al., 1994; Hernández-Rosales, 1995; Alcántara y Luna-Vega, 1997; Mayorga et al., 1998; Alcántara y Luna-Vega, 2001; Ponce-Vargas et al., 2006) los helechos y licopodios pueden o no estar incluidos. Los únicos estudios específicos sobre este grupo de plantas se han realizado en la barranca de Omitlán (Sánchez-Mejorada y Chávez, 1951) y en la Reserva de la Biosfera Barranca de Metztitlán (Cuevas y Sánchez-González, 2008). En el listado de especies vegetales del Parque Nacional Los Mármoles (PNM) sólo se citan 
cuatro familias, 10 géneros y 14 especies de licopodios y helechos (ConANP, 2007). Estos datos reflejan la carencia de estudios florísticos formales en esta área natural protegida del estado de Hidalgo. Los licopodios y helechos son parte importante de la estructura del bosque de montaña; se les encuentra en los microambientes rocosos, del suelo y del dosel arbóreo; además, participan en el balance hídrico y en la regeneración de los bosques. Por lo anterior, conocer la diversidad, distribución y dinámica de sus poblaciones es fundamental para entender los procesos de sucesión (Hill y Silander-Jr., 2001; Paciencia y Prado, 2005). Son considerados indicadores de cambios ambientales por su sensibilidad a variaciones micro-climáticas (Beukema y Van Noordwijk, 2004; Salovaara et al., 2004; Karst et al., 2005; Cárdenas et al., 2007). En adición a los servicios ambientales que representan en su hábitat natural, algunas especies de licopodios $\mathrm{y}$ helechos tienen usos directos como plantas medicinales, de ornato, elaboración de artesanías y como sustrato (Lira y Riba, 1993; Pérez-García et al., 1995; Tejero-Díez, 2007). A pesar de la importancia biológica y económica de la pteridoflora, se conoce poco sobre su riqueza, ecología y distribución tanto a nivel mundial como regional, por lo que el objetivo de la presente investigación fue proporcionar información sobre la composición taxonómica y evaluar su distribución y semejanza entre los distintos tipos de vegetación del parque nacional Los Mármoles (PNM), estado de Hidalgo.

Caracterización del área de estudio: El parque nacional
Los Mármoles (PNM) se localiza en la porción noroeste del estado de Hidalgo (figura 1), entre $20^{\circ} 45^{\prime} 39^{\prime \prime}$ y $20^{\circ} 58^{\prime} 22^{\prime}$ " de latitud norte y entre $99^{\circ} 08^{\prime} 57$ y $99^{\circ} 18^{\prime} 39^{\prime \prime}$ de longitud oeste. Tiene una superficie de 23,150 ha y ocupa parte de los municipios de Jacala de Ledesma (7,986 ha), Nicolás Flores (5,787 ha), Pacula (1,041 ha) y Zimapán (8,334 ha). La principal vía de acceso al parque es por la carretera federal número 85 que va de la ciudad de Pachuca, Hidalgo a Ciudad Valles, S.L.P.

Desde el punto de vista fisiográfico el PNM se encuentra en la intersección interna continental de la Faja Volcánica Trans-mexicana y la Sierra Madre Oriental, donde la punta norte de las sierras de Pachuca-Zimapán son las principales cadenas montañosas. El intervalo altitudinal en el PNM va de $\operatorname{los} 1,200$ a $\operatorname{los} 2,700 \mathrm{~m}$.

Las prominencias topográficas más importantes son la barranca de San Vicente, que tiene una profundidad de 600 $\mathrm{m}$ y el cerro de Cangandó con una altitud de 2,820 m. La mayoría de las rocas que afloran en el parque son de tipo sedimentario marino, constituidas por calizas y lutitas; existen afloramientos de rocas metamórficas, como las pizarras y rocas ígneas intrusivas y extrusivas. La edad de estas formaciones, varía desde el Jurásico Superior para la formación Las Trancas; del Cretácico Medio para la formación El Doctor y del Cretácico Inferior para la formación Agua Nueva; del mismo tiempo geológico son las rocas intrusivas que se introdujeron a la secuencia sedimentaria anterior (CONANP, 2007). Los suelos presentes en el parque corresponden a los

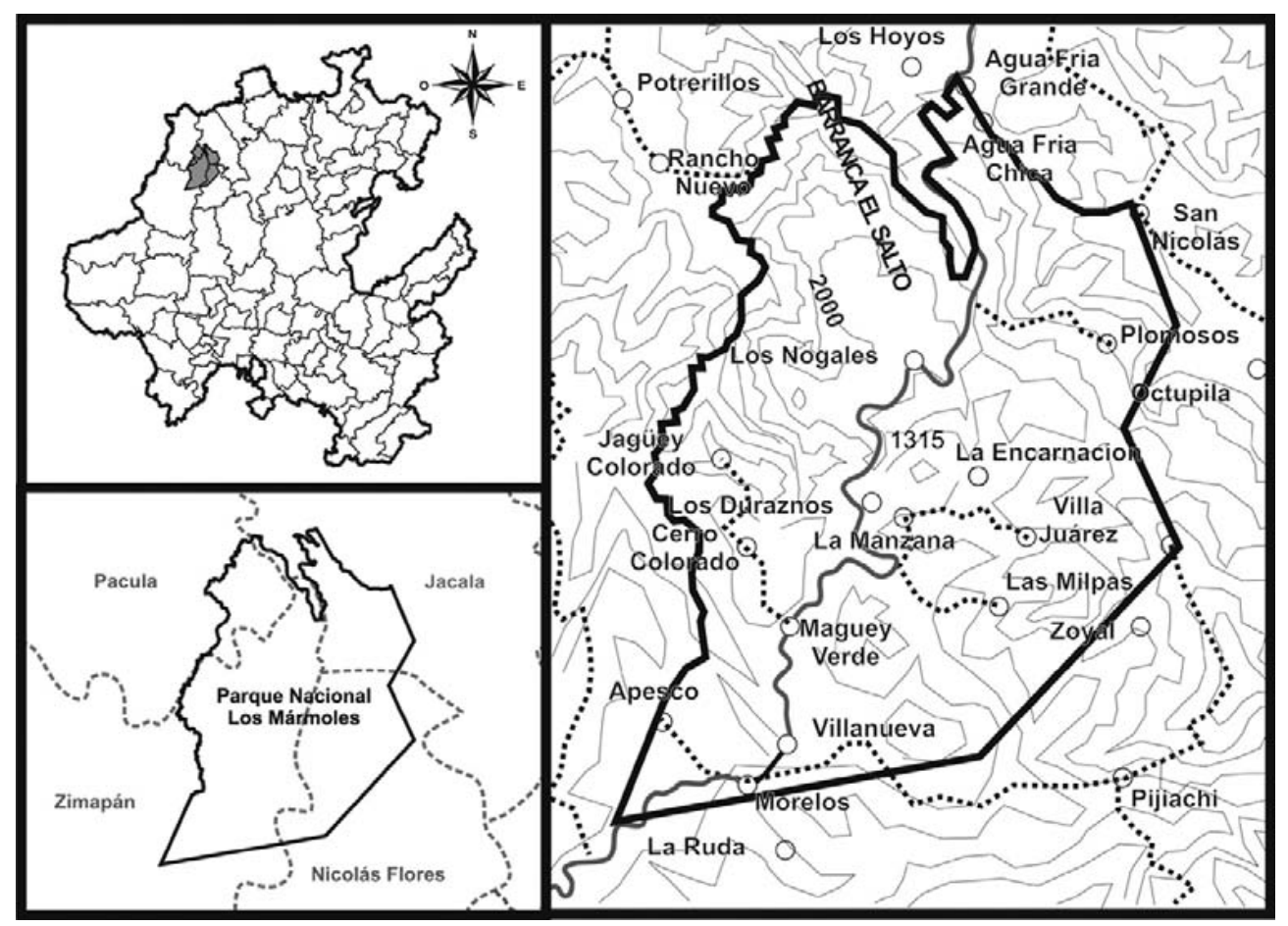

Figura 1. Localización del área de estudio (tomado de Mapas de México: www.mapas-de-mexico.com/hidalgo-state-mexico/) 
tipos de los regosoles, litosoles, luvisoles, los feozem y las rendzinas. El PNM se encuentra en la región hidrológica denominada cuenca del bajo río Pánuco. El río Moctezuma pasa al occidente del parque y recibe varios arroyos, uno de los más importantes es el de los Naranjos, que se le une cerca del poblado de Macazintla. Por la vertiente oriental fluye el río Amajac, subsidiario a su vez del río Moctezuma, el cual recibe por la margen izquierda numerosos arroyos que nacen dentro del parque, entre los más importantes están el de Barranca Seca y Rincón del Agua (ConAnp, 2007). No existen estaciones meteorológicas dentro del parque, pero de acuerdo al gradiente de altitud y la posición geográfica en el interior continental (vertiente de sotavento), presenta un clima que transita desde templado semiárido (ejemplo estación Zimapán a 1,813 m), templado subhúmedo (por ejemplo en la sierra El Doctor, Qro. a 2,730 m) hasta el semicálido subhúmedo (representado por la estación Chalpuhuacán a 1,800 $\mathrm{m})$. La temperatura anual promedio se encuentra entre los $14^{\circ} \mathrm{C}$ en la región seca, $11^{\circ} \mathrm{C}$ en la sierra y $19^{\circ} \mathrm{C}$ en la porción norte. El régimen de lluvia tiene un gradiente de 450 , 820 y cerca de $\operatorname{los} 1,500 \mathrm{~mm}$ anuales respectivamente en cada una de las estaciones, distribuida principalmente entre los meses de junio a agosto con incremento en mayo y decremento en septiembre. La temporada de sequías es de octubre a abril con leve precipitación en las zonas altas en invierno-primavera (Vargas, 1997; ConANP, 2007). Destaca en este lugar una vegetación de índole templado-subhúmedo, propia de las laderas de sotavento a mediana altitud de las sierras orientales mexicanas, compuesta por bosques de coníferas y mixtos. Las principales expresiones son: bosque de enebro, donde dominan las especies del género Juniperus (J. deppeana y J. flaccida); el bosque de enebro-encino, donde Juniperus deppeana, J. flaccida, Quercus affinis, $Q$. laeta, $Q$. mexicana y $Q$. rugosa se asocian con elementos semicálidos como Annona sp., Juglans mexicana, Bocconia arborea, Arbutus sp. y Acacia sp. El bosque de pino presenta dos asociaciones importantes: el bosque de Pinus cembroides asociado con Juniperus deppeana y el bosque de Pinus greggii, P. teocote, P. patula y P. pseudostrobus, con Crataegus mexicana y Baccharis conferta. Estos pinares pueden tener una asociación mixta con encinos como Quercus castanea, Q. crassifolia y Q. mexicana (Álvarez, 2008), acompañados de elementos más xerófilos como Opuntia spp., Buddleja, Arbutus y Juniperus. Otros tipos de vegetación menos comunes en el parque son: el matorral espinoso, con presencia de especies como Acacia berlandieri, Dalea bicolor, Mimosa aculeaticarpa, Caesalpinia mexicana, Senna racemosa y Karwinskia sp., y la selva baja caducifolia, donde las especies más comunes en el estrato arbóreo son Cordia boissieri, Acacia sp., Bursera sp., Proposis laevigata, Leucaena sp., Sideroxilon sp., Casimiroa sp. y Brosimum sp. La vegetación secundaria de matorral y pastizal es frecuente, como producto de las actividades agropecuarias en el área (CONANP, 2007).

\section{Materiales y métodos}

El trabajo de campo consistió en la exploración y recolección exhaustiva de ejemplares, de acuerdo al método propuesto por Lorea y Riba (1990), en cada uno de los tipos de vegetación existentes de los municipios de Jacala de Ledezma, Nicolás Flores, Pacula y Zimapán, que forman el PNM. La elección de los sitios para la recolección de ejemplares fue preferencial; se basó en el reconocimiento cartográfico y fisonómico de los diferentes tipos de vegetación y se hicieron lo más distante posible de los centros de población (como un criterio indirecto cualitativo que indica menor perturbación humana). La recolección se realizó durante 21 meses consecutivos, período en el que se exploraron nueve diferentes ambientes, en un total de 34 sitios. Cada sitio fue explorado al menos en tres ocasiones: una vez durante la temporada de pre-lluvias y dos al finalizar las lluvias.

Determinación taxonómica.- Los ejemplares se determinaron con base en el trabajo de Mickel y Smith (2004) y se compararon con material depositado en el herbario de la Facultad de Estudios Superiores Iztacala de la Universidad Nacional Autónoma de México. Los ejemplares de referencia se integraron a la colección del herbario del Centro de Investigaciones Biológicas de la Universidad Autónoma del Estado de Hidalgo (Herbario HGOM) y una copia se donará al herbario nacional (MEXU), con sede en el Instituto de Biología de la UNAM. Los taxones fueron organizados en una lista de acuerdo al sistema de clasificación propuesto por Smith et al. (2006).

El patrón de distribución de las especies de helechos y licopodios en los diferentes tipos de vegetación se estimó en forma numérica, con datos de presencia/ausencia, mediante el análisis de agrupamiento, con el programa de cálculo PC-ORD (McCune y Mefford, 1999). Se eligió el índice de Sørensen para definir la semejanza entre los grupos, por ser de los más robustos para datos ecológicos. Se utilizó el ligamiento promedio como método de unión de grupos (UPGMA), ya que introduce relativamente poca distorsión en la distancia entre agrupamientos con respecto a la matriz de distancias original y evita el efecto de encadenamiento generado con otros métodos de unión. Los resultados se presentan en un dendrograma en el que la medida de distancia se transformó a la función objetiva de Wishart (expresada como porcentaje de información remanente), que evita que la unión de dos grupos ocurra a una distancia menor que en una fusión previamente establecida (McCune y Mefford, 1999). Para determinar el número de grupos en el dendrograma se eligió un nivel de corte que considerara un compromiso entre la pérdida de información y la simplificación de un número de unidades de vegetación interpretables desde un punto de vista natural (McCune y Grace, 2002). 
Cuadro 1. Número de taxa por familias, géneros y especies de Pteridophytas y grupos afines del Parque Nacional los Mármoles

\begin{tabular}{lccc}
\hline Pteridobiontes & Familias & Géneros & Especies \\
\hline Polypodiophyta & 11 & 28 & 65 \\
Lycopodiophyta & 1 & 1 & 6 \\
$\begin{array}{l}\text { Porcentaje con respecto al } \\
\text { citado por Mickel y Smith } \\
\text { (2004) para el estado de }\end{array}$ & $48 \%$ & $43 \%$ & $27 \%$ \\
Hidalgo & & & \\
\end{tabular}

\section{Resultados}

Pteridoflora del PNM. Se recolectaron en total 520 ejemplares de helechos y licopodios. Una vez determinados se encontraron 11 familias, 28 géneros y 65 especies de helechos y una familia monogenérica con seis especies de licopodios (Cuadro 1 y Apéndice 1). Las familias con mayor número de géneros fueron Pteridaceae con 12 (41\%), Polypodiaceae con cuatro (14\%), Woodsiaceae, Dryopteridaceae y Blechnaceae, con dos géneros (7\%), las familias restantes están representadas sólo por un género. Las familias con mayor número de especies fueron: Pteridaceae con 28 (41\%), Polypodiaceae con 19 (27\%), Selaginellaceae con seis $(8 \%)$, Dryopteridaceae con cuatro $(6 \%)$ y Aspleniaceae con tres $(4 \%)$. Las familias restantes, con una o dos especies, representaron el $14 \%$, en conjunto. Los géneros con mayor número de especies fueron: Polypodium, con 13 (18\%); Cheilanthes, con siete (10\%); Selaginella, con seis (8\%); Adiantum y Notholaena con cuatro (6\%). Los géneros restantes presentaron entre una y tres especies. Las especies que se pueden considerar como generalistas, por tener

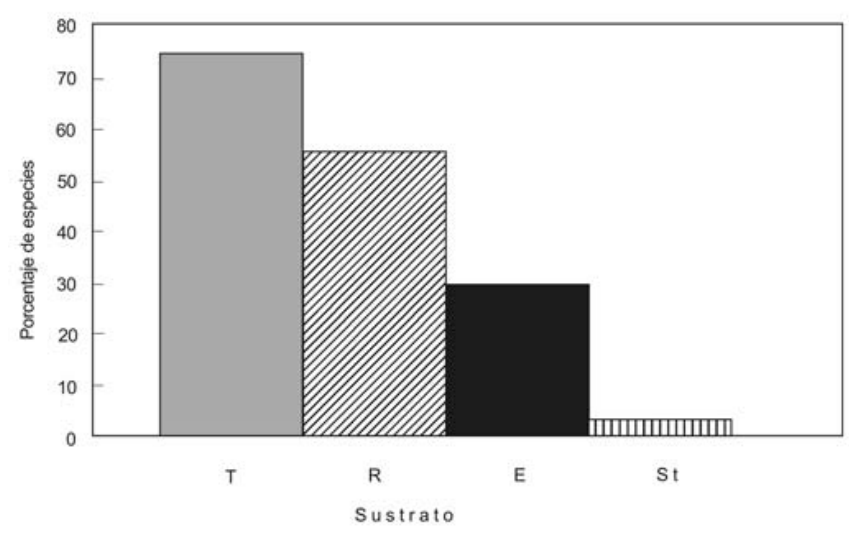

Figura 2. Sustrato en el que crecen los licopodios y helechos. T: terrestre, R: rupícola, E: epífita, St: sobre tronco caído. Se citan 71 especies, pero algunas se encontraron hasta en dos tipos de sustrato de crecimiento.

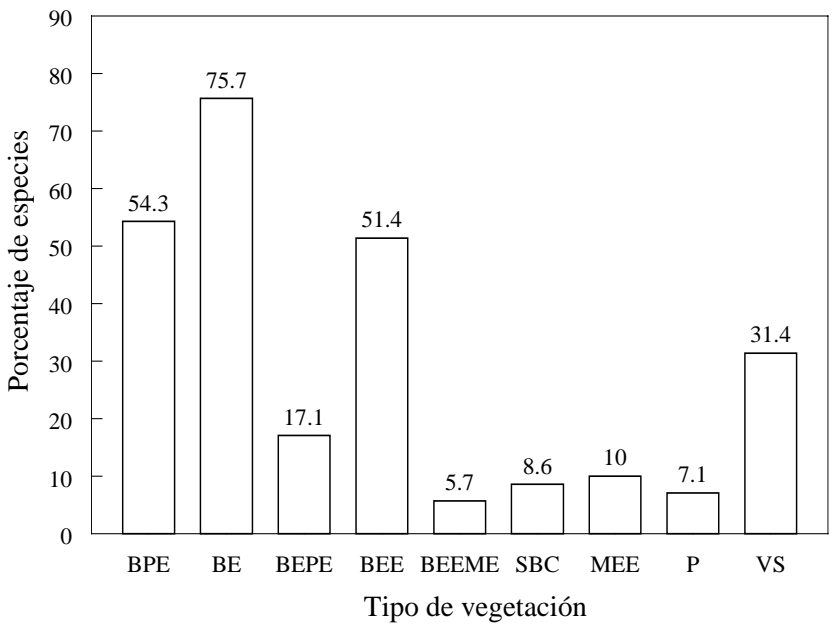

Figura 3. Porcentaje de especies de helechos y licopodios en cada tipo de vegetación

la más amplia distribución dentro del PNM fueron: Cheilanthes notholaenoides con una frecuencia de presencia de $85 \%$, Asplenium monanthes con $62 \%$, Polypodium polypodiodes var. aciculare con 47\%, Mildella fallax y Pleopeltis polylepis var. polylepis con 44\%, Polypodium martensii con $41 \%$, Cheilanthes bonariensis con 38\%, Asplenium resiliens, Adiantum andicola y Llavea cordifolia con 35\%, las demás especies presentaron un porcentaje de constancia menor de $32 \%$. Los sustratos más comunes en los que se recolectaron los ejemplares de licopodios y helechos fueron: en tierra (74\%), rupícolas (55\%), epífitas (29\%) y los que crecen sobre troncos caídos (3\%, figura 2).

Nuevos registros para el estado de Hidalgo. Se consideraron cinco especies y dos variedades como nuevos registros para el estado de Hidalgo, dado que no estaban mencionadas en el trabajo de Mickel y Smith (2004): Anemia mexicana var. makrinii, Argyrochosma palmeri, Cheilanthes spiculata,

Cuadro 2. Comparación fisiográfica y de riqueza florística entre tres sitios propios de la región de la Sierra Madre Oriental y la Faja Volcánica Trans-mexicana

\begin{tabular}{llccc}
\hline Localidad & $\begin{array}{l}\text { Intervalo de } \\
\text { altitud }(\mathrm{m})\end{array}$ & $\begin{array}{c}\text { Área } \\
\text { (ha) }\end{array}$ & $\begin{array}{c}\text { Número de } \\
\text { especies }\end{array}$ & $\begin{array}{c}\mathrm{RF}=\mathrm{S} / \mathrm{Ln} \mathrm{A} \\
\text { spp/ha }\end{array}$ \\
\hline PNM, Hidalgo & $1,300-2,950$ & 23,150 & 71 & 6.97 \\
$\begin{array}{l}\text { Barranca de } \\
\text { Omitlán, Hidalgo }\end{array}$ & $2,080-3,048$ & 2,000 & 52 & 6.84 \\
$\begin{array}{l}\text { Tlatlauquitepec, } \\
\text { Puebla }\end{array}$ & $800-2,000$ & 24,600 & 66 & 6.53 \\
\end{tabular}




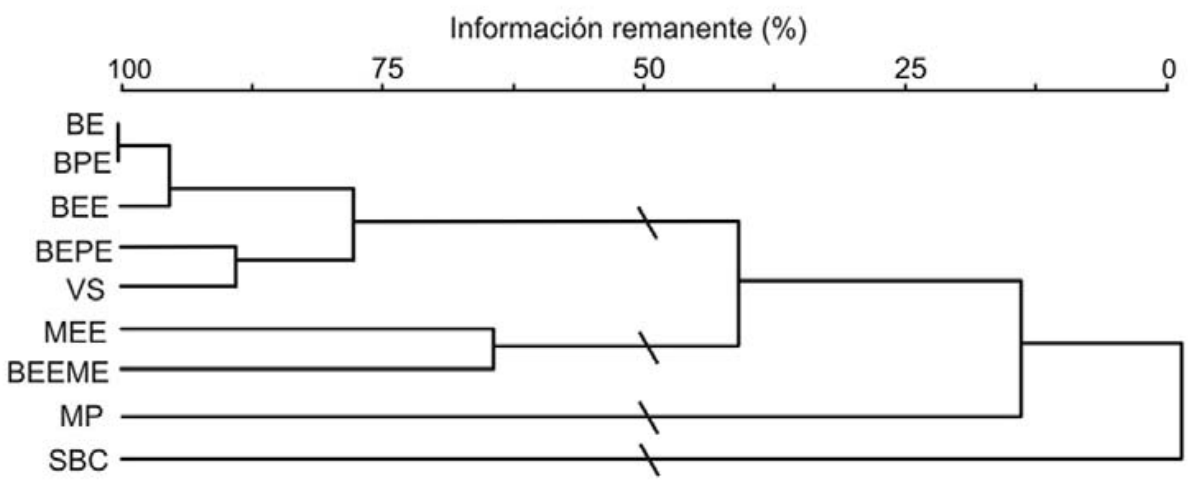

Figura 4. Dendrograma del análisis de agrupamiento que muestra las relaciones jerárquicas entre los tipos de vegetación, de acuerdo a la composición de especies de licopodios y helechos. Cada rama del dendrograma está etiquetada con el tipo de vegetación correspondiente. La escala vertical es la función objetiva de Wishart e indica el porcentaje de información presente a medida que los grupos se fusionan ( $\backslash$ : nivel de corte). Bosque de encino (BE), bosque de pino-encino (BPE), bosque de encino-enebro (BEE), bosque de encino-pino-enebro (BEPE), bosque de encino-enebro-matorral espinoso (BEEME), matorral espinoso-enebro (MEE), selva baja caducifolia (SBC), pastizal (MP), vegetación secundaria (VS).

Pecluma alfredii var. cupreolepis, Polypodium puberulum, Selaginella sartorii y $S$. wrightii (Apéndice 1).

Distribución de las especies en los diferentes tipos de vegetación. Se delimitaron nueve tipos de vegetación, con base en las especies dominantes: bosque de encino (BE), bosque de pino-encino (BPE), bosque de encino-enebro (BEE), bosque de encino-pino-enebro (BEPE), bosque de encinoenebro-matorral espinoso (BEEME), matorral espinosoenebro (MEE), selva baja caducifolia (SBC), pastizal (P) y vegetación secundaria (VS) (figura 3). El bosque de encino y los otros tipos de vegetación en donde los encinos son codominantes, se ven favorecidos por la presencia de helechos y licopodios. En el bosque de encino destacan las especies epífitas, debido a que los encinos son buenos hospederos y a los micro-ambientes húmedos que esta comunidad ocupa (presencia de cárcavas y cañadas húmedas).

Muy pocas especies se encuentran, en cambio, en el resto de los ambientes secos como el semiárido y el cálido, en tales lugares ocupan sitios protegidos como la base de rocas y cárcavas de arroyos. El carácter indicador de los helechos y licopodios se pone de manifiesto al realizarse un ensayo de similitud entre los ambientes (figura 4). Cuatro grupos permitieron inferir las relaciones entre los tipos de vegetación: el primer grupo está formado por especies características de vegetación templada sub-húmeda donde dominan encinos, pinos y enebros; el segundo grupo consta de especies que se encuentran en vegetación de zonas semiáridas con elementos de matorral xerófilo; el tercer grupo de helechos y licopodios tiene preferencia por el pastizal, un tipo de vegetación aislado y escaso en el parque, sobre sustrato de roca ígnea y que, en términos operativos, puede considerarse como un ambiente seco; el cuarto grupo de especies se encuentra en vegetación con afinidad cálido sub-húmeda.

\section{Discusión}

Riqueza florística. Las comunidades ecológicas difieren entre sí, entre otros aspectos, en cuanto al número y tipo de especies que incluyen. Aplicando el índice de biodiversidad taxonómica (IB), definido por el número de especies dividido entre el logaritmo natural del área en ha $(\mathrm{IB}=\mathrm{S} / \mathrm{Ln} \mathrm{A}$, donde $\mathrm{S}$ será el número de las especies registradas y $\mathrm{A}$ el tamaño de área, Squeo et al., 1998; Ponce et al., 2002), se encontró que en el PNM la riqueza es de siete especies de licopodios y helechos por ha. Este índice comparado con el de otras áreas de las misma zona fisiográfica con estudios pteridoflorísticos (Sánchez-Mejorada y Chávez, 1951; Cerón-Carpio et al., 2006) (Cuadro 2), permite observar que la diferencia es muy reducida aún considerando que en el PNM una buena parte del terreno es de índole sub-húmedo comparado con las otras dos localidades. Es bien sabido que los climas sub-húmedos no favorecen la presencia de licopodios y helechos (Moran, 2004; Tejero-Díez y ArreguínSánchez, 2004), por lo que la elevada riqueza de especies debe estar relacionada con la gran heterogeneidad fisiográfica del PNM (donde no existen zonas de valles ni cuencas), con el amplio intervalo altitudinal (mayor de 1,500 m) y con la presencia de numerosas cañadas y arroyos, donde coexisten diferentes comunidades vegetales (ConANP, 2007). Las familias que presentaron la mayor riqueza a nivel de género en el PNM (Pteridaceae, Polypodiaceae, Woodsiaceae, Dryopteridaceae y Blechnaceae), se citan también como las más diversas en los bosques de pino-encino de Omitlán (Sánchez-Mejorada y Chávez, 1951) y en los bosques mesófilos de montaña de los municipios de Tlanchinol, Tenango de Doria, Molocotlán, Eloxochitlán, Tlahuelompa y Lolotla (Luna-Vega et al., 1994; Alcántara y Luna-Vega, 1997; Mayorga et al., 1998; Alcántara y Luna-Vega, 2001; Ponce- 
Vargas et al., 2006). Los géneros con más de cinco especies los constituyen Pleopeltis [sensu Windham (1993), que considera a las especies de Polypodium con hojas escamosas poikilohídricas] (9 spp., $12.8 \%$ ), Cheilanthes (7 spp., $10 \%$ ), Polypodium (s.s.) y Selaginella (principalmente del grupo isófilo) con seis especies $(8.6 \%)$. En total estos géneros contienen el $40 \%$ de las especies del lugar, conjunto de taxones que reflejan claramente la zona ecológica templado sub-húmeda a semiárida de la localidad estudiada, parecida a la de otras sierras del interior continental (Cartujano et al., 2002; Cabrera-Luna y Gómez-Sánchez, 2005; CerónCarpio et al., 2006; Medina y Tejero-Díez, 2006; Vázquez et al., 2006).

El hecho de que el estado de Hidalgo ha recibido poca atención botánica y de que la mayoría de los estudios florísticos han tenido como objetivo principal a las gimnospermas y angiospermas, se pone de relieve al analizar los resultados de distintos estudios realizados en bosques templados húmedos (bosques mesófilos de montaña). Luna-Vega et al. (1994), Alcántara y Luna-Vega (1997), Mayorga et al. (1998), Alcántara y Luna-Vega (2001) y Ponce-Vargas et al. (2006), citan números muy bajos de especies de helechos y licopodios (entre 32 y 39) en relación con bosques mesófilos de la misma provincia florística (Tejero-Díez y Mickel, 2004), lo cual explica la carencia de un buen inventario pteridológico para el estado. Por lo tanto, no fue sorpresa encontrar siete especies del PNM como nuevos registros para el estado. Algunas de estas especies son de amplia distribución en México, por lo que era de esperarse su presencia en Hidalgo. Otras, en cambio, son de distribución restringida como Cheilanthes spiculata que sólo se había recolectado en el Distrito Federal y Polypodium puberulum citado en los estados de Chiapas, Guerrero, Oaxaca y Puebla (Mickel y Smith, 2004). Aunque este patrón de distribución puede deberse simplemente a la carencia de estudios florísticos en otros estados de la República Mexicana, existe la posibilidad de que las especies tengan distribución disyunta (Espinosa-Organista et al., 2002).

Los resultados de la presente investigación indican que hay ensambles de especies de licopodios y helechos, característicos de distintos tipos de vegetación en el PNM; esta información es relevante pues como lo han mencionado distintos autores este grupo de plantas puede ser utilizado como indicador ambiental y del estado de conservación de los ecosistemas (Beukema y Van Noordwijk, 2004; Salovaara et al., 2004; Karst et al., 2005).

Como en todas las áreas naturales protegidas de México, en el parque nacional Los Mármoles la vegetación y los recursos naturales en general están en peligro. Los problemas más graves son los asentamientos humanos, la extracción de mármol, la tala y el cambio de uso del suelo hacia campos de cultivo o pastizales (Álvarez y Sánchez-González, 2007). Recientemente, en un estudio realizado por la CoNANP (2007), se plantearon algunos criterios para la reducción de la extensión territorial del PNM y el cambio de denominación a Área Natural de Protección de Flora y Fauna. Las consecuencias de esta reducción y probable re-categorización deben ser analizadas con detenimiento, con base en estudios formales que consideren aspectos económicos, sociales y culturales, para así preservar los recursos y procesos naturales en el PNM.

\section{Agradecimientos}

El presente estudio se realizó con fondos del proyecto PROMEP titulado "Estudio florístico, biogeográfico y sinecológico del Parque Nacional Los Mármoles" y con el proyecto FOMIX Hidalgo 43761. Alin N. Torres-Díaz y dos dictaminadores anónimos auxiliaron con sus observaciones a mejorar este manuscrito.

\section{Literatura citada}

Alcántara A.O. y Luna-Vega I. 1997. Florística y análisis biogeográfico del bosque mesófilo de montaña de Tenango de Doria, Hidalgo, México. Anales del Instituto de Biología de la Universidad Nacional Autónoma de México, Serie Botánica 68:57-106.

Alcántara A.O. y Luna-Vega I. 2001. Análisis florístico de dos áreas con bosque mesófilo de montaña en el estado de Hidalgo, México: Eloxochitlán y Tlahuelompa. Acta Botánica Mexicana 54:51-87.

Álvarez Z.E. 2008. El género Quercus en el Parque Nacional Los Mármoles, estado de Hidalgo y morfología foliar de Quercus laeta Liebm. Tesis de Licenciatura, Universidad Autónoma del Estado de Hidalgo. Pachuca, Hidalgo. 93 pp.

Álvarez Z.E. y Sánchez-González A. 2007. Los encinos del estado de Hidalgo. Herreriana, Revista de Divulgación de la Ciencia 3:22.

Arreguín-Sánchez M.L., Fernández-Nava R. y Rodríguez J.A. 1996. Pteridofitas en el estado de Querétaro, México y su ubicación ecológica. Polibotánica 3:82-92.

Beukema H. y Van Noordwijk M. 2004. Terrestrial pteridophytes as indicators of a forest-like environment in rubber production systems in the lowlands of Jambi, Sumatra. Agriculture, Ecosystems and Environment 104:63-73.

Cabrera-Luna.J.A. y Gómez-Sánchez.M. 2005. Análisis florístico de la Cañada, Querétaro, México. Boletín de la Sociedad Botánica de México 77:35-50.

Calderón de Rzedowski G. y Rzedowski J. 2001. Flora fanerogámica del Valle de México. Segunda Edición. Instituto de Ecología y Comisión Nacional para el Conocimiento y Uso de la Biodiversidad. Pátzcuaro, Michoacán, México. 1406 pp.

Cárdenas G.G., Halme K.J. y Tuomisto H. 2007. Riqueza y distribución ecológica de especies de pteridofitas en la zona del Río Yavarí-Mirín, Amazonía Peruana. Biotropica 39:637-646.

Cartujano S., Zamudio S., Alcántara O. y Luna-Vega I. 2002. El bosque mesófilo de montaña en el municipio de Landa de Matamoros, Querétaro. Boletín de la Sociedad Botánica de México 70:13-43.

Cerón-Carpio A.B., Arreguín-Sánchez M.L. y Fernández-Nava R. 2006. Listado con anotaciones de las pteridofitas del municipio 
de Tlatlauquitepec, Puebla, México y distribución de las especies en los diferentes tipos de vegetación. Polibotánica 21:45-60.

Challenger A. 1998. Utilización y conservación de los ecosistemas terrestres de México. Pasado, presente y futuro. Comisión Nacional para el Conocimiento y Uso de la Biodiversidad. México, D.F. 847 pp.

Conanp [Comisión Nacional de Áreas Naturales Protegidas]. 2007. Estudio previo justificativo para la modificación del decreto por el que se pretende re-categorizar el Parque Nacional Los Mármoles como Área de Protección de Flora y Fauna. Secretaria del Medio Ambiente y Recursos Naturales. México, D.F. 89 pp.

Cuevas H.A.L. y Sánchez-González A. 2008. Las pteridofitas de la reserva de la biosfera Barranca de Meztitlán, estado de Hidalgo, México. En: Simposio Internacional sobre Flora Silvestre de Zonas Áridas: 282-295. Centro de Investigaciones Biológicas del Noroeste, S.C., La Paz, Baja California Sur. México.

Espinosa-Organista D., Morrone J.J., Llorente-Bousquets J. y Flores-Villela O. 2002. Introducción al análisis de patrones en biogeografía histórica. Las Prensas de Ciencias. Facultad de Ciencias, UNAM. México, D.F. 133 pp.

Hernández-Rosales M.R. 1995. Estudio florístico-fanerogámico del parque nacional El Chico, estado de Hidalgo. Tesis de Licenciatura, Escuela Nacional de Estudios Profesionales Iztacala, Universidad Nacional Autónoma de México. Tlalnepantla, Estado de México. 73 pp.

Hill J.D. y Silander-Jr. J.A. 2001. Distribution and dynamics of two ferns: Dennstaedtia punctilobula (Dennstaedtiaceae) and Thelypteris noveboracensis (Thelypteridaceae) in a northeast mixed hardwoods-hemlock forest. American Journal of Botany 88:894-902

Karst J., Gilbert B. y Lechowicz M.J. 2005. Fern community assembly: the roles of chance and the environment at local and intermediate scales. Ecology 86:2473-2486.

Lira R. y Riba R. 1984. Aspectos fitogeográficos y ecológicos de la flora pteridófita de la sierra de Santa Marta, Veracruz, México. Biótica 9:451-467.

Lira R. y Riba R. 1993. Las pteridofitas (helechos y plantas afines) de México. Revista de la Sociedad Mexicana de Historia Natural (Volumen Especial) 44:99-108.

Lorea F. y Riba R. 1990. Guía para la recolección y preparación de ejemplares para herbario de pteridofitas. Consejo Nacional de la Flora de México, A. C. México, D.F. 12 pp.

Lorea-Hernández F. y Velázquez-Montes E. 1998. Pteridofitas: lista de los taxa y su distribución geográfica en la entidad. En: Diego-Pérez N. y Fonseca R. Ma. Eds. Estudios florísticos en Guerrero No. 9. Facultad de Ciencias, Universidad Nacional Autónoma de México. México, D.F. 83 pp.

Luna-Vega I., Ocegueda C.S. y Alcántara A.O. 1994. Florística y notas biogeográficas del bosque mesófilo de montaña del municipio de Tlanchinol, Hidalgo, México. Anales del Instituto de Biología de la Universidad Nacional Autónoma de México, Serie Botánica 65:31-62.

Mayorga S.R., Luna-Vega I. y Alcántara A.O. 1998. Florística del bosque mesófilo de montaña de Molocotlán, Molango Xochicoatlán, Hidalgo, México. Boletín de la Sociedad Botánica de México 63:101-119.

McCune B. y Mefford M.J. 1999. PC-ORD. Multivariate Analysis of Ecological Data. Version 4 for Windows. MjM Software Design. Gleneden Beach, Oregon. 237 pp.

McCune B. y Grace J.B. 2002. Analysis of ecological communities.
MjM Software Design. Gleneden Beach, Oregon. 300 pp.

Medina L.J.G. y Tejero-Díez J.D. 2006. Flora y vegetación del Parque Estatal Atizapán-Valle Escondido estado de México, México. Polibotánica 21:1-43.

Mickel J.T. 1992. Pteridophytes. En: McVaugh R. Ed. Flora NovoGaliciana. A descriptive account of the vascular plants of Western Mexico: 120-467. The University of Michigan Herbarium, Ann Arbor, Michigan.

Mickel J.T. y Smith A.R. 2004. The Pteridophytes of Mexico. Memoirs of the New York Botanical Garden. NYBG press. $1054 \mathrm{pp}$.

Moran, R.C. 2004. A natural history of ferns. Timber Press. Portland \& Cambridge. 301 pp.

Paciencia M.L.B. y Prado J. 2005. Effects of forest fragmentation on pteridophyte diversity in a tropical rain forest in Brazil. Plant Ecology 180:87-104.

Pérez-García B., Riba R. y Reyes-Jaramillo I. 1995. Helechos mexicanos: formas de crecimiento, hábitat y variantes edáficas. Contactos 11:22-27.

Ponce M., Mehltreter K. y de la Sota E.R. 2002. Análisis biogeográfico de la diversidad pteridofítica en Argentina y Chile continental. Revista Chilena de Historia Natural 75:703-717.

Ponce-Vargas A., Luna-Vega I., Alcántara-Ayala O. y Ruiz-Jiménez C.A. 2006. Florística del bosque mesófilo de montaña de Monte Grande, Lolotla, Hidalgo, México. Revista Mexicana de Biodiversidad 77:177-190.

Riba R., L. Pacheco A. Valdes y Sandoval Y. 1996. Pteridoflora del estado de Morelos, México. Lista de familias, géneros y especies. Acta Botánica Mexicana 37: 45-65

Salovaara K.J, Cárdenas G.G. y Tuomisto H. 2004. Forest classification in an Amazonian rainforest landscape using pteridophytes as indicator species. Ecography 27:689-700.

Sánchez-Mejorada H. y Chávez C. 1951. Breves notas sobre las pteridofitas de la barranca de Omitlán, Hidalgo. Boletín de la Sociedad Botánica de México 12:28-36.

Smith A.R., Pryer K.M., Schuettpelz E., Korall P., Schneider H. y Wolf P. 2006. A classification for extant ferns. Taxon 55:705-731.

Squeo F., Cavieres L., Arancio G., Novoa J.E., Matthei O., Marticorena C., Rodríguez R., Arroyo M.T.K. y Muñoz M. 1998. Biodiversidad de la flora vascular en la región de Antofagasta, Chile. Revista Chilena de Historia Natural 71:571-591.

Tejero-Díez J.D. 1998. Pteridoflora del occidente del estado de México. Tesis de Maestría. Facultad de Ciencias, Universidad Nacional Autónoma de México, México, D.F. 142 pp.

Tejero-Díez J.D. y Arreguín-Sánchez M.L. 2004. Lista con anotaciones de los pteridófitos del estado de México, México. Acta Botánica Mexicana 69:1-82.

Tejero-Díez, J.D. y Mickel J.T. 2004. Pteridofitas. En: GarcíaMendoza, A.J., M.J. Ordoñez y M. Briones-Salas (Eds.). Biodiversidad de Oaxaca: 121-139. Instituto de Biología,Universidad Nacional Autónoma de México, Fondo oaxaqueño para la conservación de la naturaleza y World Wildlife Fund. México.

Tejero-Díez J.D. 2007. La riqueza florística del estado de México: licopodios y helechos. Adumbrationes Ad Summae Editionem 27:1-32.

Vargas M.F. 1997. Parques Nacionales de México. Aspectos físicos, sociales, legales, administrativos, recreativos, biológicos, culturales, situación actual y propuestas en torno a los parques nacionales de México. Instituto Nacional de Ecología. México, D.F. 
RAMÍREZ-CRUZ ET AL.

Vázquez T.M., Campos J.J. y Cruz P.A. 2006. Los helechos y plantas afines del bosque mesófilo de montaña de Banderilla, Veracruz, México. Polibotánica 22:63-77.

Villaseñor J.L. 2003. Diversidad y distribución de las Magno- liophyta de México. Interciencia 28:160-167.

Windham M.D. 1993. New taxa and nomenclatural changes in the North American fern flora. Contributions from the University of Michigan Herbarium 19:31-61

Recibido: 14 de agosto de 2008

Aceptado: 29 de enero de 2009 
Apéndice 1. Listado de las especies de licopodios y helechos del PNM, estado de Hidalgo de acuerdo a Smith et al. (2006). Entre paréntesis se encuentra la referencia de colecta con las iníciales del primer autor.

\section{LYCOPODIOPHYTA}

\section{SELAGINELLOPSIDA}

Selaginellaceae

1. Selaginella extensa Underw. (SRC 328)

2. Selaginella lepidophylla (Hook. \& Grev.) Spring (SRC 329)

3. Selaginella pallescens (C. Presl) Spring (SRC 333)

4. Selaginella pulcherrima Liebm. ex E. Fourn. (SRC 335)

5. Selaginella sartorii Hieron.* (SRC 327)

6. Selaginella wrightii Hieron. * (SRC 222)

BE
SBC
BE, BEE, MEE, MP
BEE
BE, MP
BEE

\section{POLYPODIOPHYTA}

PSILOTOPSIDA

Ophioglossaceae

7. Botrychium virginianum (L.) Sw. (SRC 115)

BE, BPE

\section{EQUISETOPSIDA}

Equisetaceae

8. Equisetum myriochaetum Schltdl. \& Cham. (SRC 158)

\section{POLYPODIOPSIDA}

\section{SCHIZAEALES}

Anemiaceae

9. Anemia adiantifolia (L.) Sw. (SRC 320)

10. Anemia mexicana Klotzsch var. makrinii (Maxon) Mickel* (SRC 324)

\section{POLYPODIALES}

Dennstaedtiaceae

11. Pteridium aquilinum (L.) Kuhn var. feei (W. Schaffn. ex Fée) Maxon ex Yunck. (SRC 179) Pteridaceae

12. Adiantum andicola Liebm. (SRC 54)

13. Adiantum capillus-veneris L. (SRC 61)

14. Adiantum poiretii Wikstr. (SRC 65)

15. Adiantum tenerum Sw. (SRC 66)

16. Argyrochosma formosa (Liebm.)Windham (SRC 155) (SRC 109)

17. Argyrochosma incana (C. Presl) Windham (SRC 291)

18. Argyrochosma palmeri (Baker) Windham* (SRC 205)

19. Astrolepis crassifola (Houlston \& T. Moore) D. M. Benham \& Windham (SRC 90)

20. Astrolepis integerrima (Hook.) D. M. Benham \& Windham (SRC 99)

21. Astrolepis laevis (M. Martens \& Galeotti) Mickel (SRC 92)

22. Bommeria ehrenbergiana (Klotzsch) Underw. (SRC 112)

23. Cheilanthes bonariensis (Willd.) Proctor (SRC 122)

24. Cheilanthes cucullans Fée (SRC 337)

25. Cheilanthes leucopoda Link (SRC 131)

26. Cheilanthes marginata Kunth (SRC 298)

27. Cheilanthes myriophylla Desv. (SRC 133)

28. Cheilanthes notholaenoides (Desv.) Maxon ex Weath. (SRC 146)

29. Cheilanthes spiculata Mickel * (SRC 302)

30. Cheiloplecton rigidum (Sw.) Fée var. rigidum (SRC 289)

31. Llavea cordifolia Lag. (SRC 162)

32. Mildella fallax (M. Martens \& Galeotti) G. L. Nesom (SRC 175)

33. Notholaena aschenborniana Klotzsch (SRC 286)

34. Notholaena candida (M. Martens \& Galeotti) Hook. (SRC 280)
BE, BEE, BPE

BE, BEE, BPE, BEPE, VS

$B E, B E E, B P E$

BE, BEE, BPE, BEPE

BE

$B E, B E E, B P E, V S$

$B E, B P E$

$B E, M P$

BE, BPE, MEE, BEEME

$B E, B E E, B P E, V S$

BE, BEE, BPE, BEPE, VS VS

BE, BPE, MP, BEPE, VS BEE, SBC MEE

BE, BPE, BEPE, VS $M P, B E P E, V S$

BE, BEE, BPE, MEE, BEPE, VS, BEEME

$B E$

$B E, B E E$

BE, BEE, BPE, VS

$B E, B E E, B P E, B E P E$, VS $B E, B E E$

BEE, SBC, MEE, VS 
35. Notholaena galeottii Fée (SRC 287)

36. Notholaena sulphurea (Cav.) J. Sm. (SRC 281)

37. Pellaea ovata (Desv.) Weath. (SRC 170)

38. Pityrogramma trifoliata (L.) R. M. Tryon (SRC 178)

39. Pteris cretica L. (SRC 169)

Aspleniaceae

40. Asplenium exiguum Bedd. (SRC 325)

41. Asplenium monanthes L. (SRC 67)

42. Asplenium resiliens Kunze (SRC 86)

Thelypteridaceae

43. Thelypteris kunthii (Desv.) C. V. Morton (SRC 182)

44. Thelypteris pilosula (Klotzsch \& H. Karst. ex Mett.) R. M. Tryon (SRC 301)

Woodsiaceae

45. Cystopteris fragilis (L.) Bernh. (SRC 117)

46. Woodsia aff. mollis (Kaulf.) J. Sm. (SRC 317)

Blechnaceae

47. Blechnum appendiculatum Willd. (SRC 336)

48. Woodwardia spinulosa M. Martens \& Galeotti (SRC 181)

Dryopteridaceae

49. Dryopteris cinnamomea (Cav.) C. Chr. (SRC 156)

50. Dryopteris pseudofilix-mas (Fée) Rothm. (SRC 155)

51. Dryopteris wallichiana (Spreng.) Hyl. (SRC 157)

52. Elaphoglossum petiolatum (Sw.) Urb. (SRC 322)

Polypodiaceae

53. Pecluma alfredii (Rosenst.) M. G. Prince var. cupreolepis (A. M. Evans) A. R. Sm. * (SRC 312)

54. Pecluma plumula (Humb. \& Bonpl. ex Willd.) M. G. Price (SRC 305)

55. Phlebodium pseudoaureum (Cav.) Lellinger (SRC 293)

56. Pleopeltis crassinervata (Fée) T. Moore (SRC 260)

57. Pleopeltis mexicana (Fée) Mickel \& Beitel (SRC 264)

58. Pleopeltis polylepis (Roemer ex Kunze) T. Moore var. polylepis (SRC 269)

59. Polypodium arcanum Maxon var. septentrionale Mickel (SRC 193)

60. Polypodium fraternum Schltdl. \& Cham. (SRC 185)

61. Polypodium ${ }^{1}$ furfuraceum Schltdl. \& Cham. (SRC 197)

62. Polypodium ${ }^{1}$ guttatum Maxon (SRC 207)

63. Polypodium ${ }^{1}$ lepidotrichum (Fée) Maxon (SRC 316)

64. Polypodium martensii Mett. (SRC 216)

65. Polypodium ${ }^{1}$ plebeium Schltdl. \& Cham. (SRC 189)

66. Polypodium plesiosorum Kunze (SRC 218)

67. Polypodium ${ }^{1}$ polypodiodes (L.) Watt var. aciculare Weath. (SRC 233)

68. Polypodium puberulum Schltdl. \& Cham. * (SRC 194)

69. Polypodium rhodopleuron Kunze (ASG 2400)

70. Polypodium subpetiolatum Hook. (SRC 244)

71. Polypodium ${ }^{1}$ thyssanolepis A. Braun ex Klotzsch (SRC 249)
BEE, VS, BEEME

$B E, B E E, B P E, V S, B E E M E$

$B E, B E P E$

SBC

$B E$

BE

$B E, B E E, B P E, B E P E, V S$

$B E, B E E, B P E$

$\mathrm{BE}, \mathrm{SBC}, \mathrm{BPE}$

$\mathrm{BE}$

BE, BPE
BPE

BE, BPE

BPE

BPE

BPE

BPE

$B E, B P E$

$B E, B E E, B P E$

BE, BEE

BE, BEE, BPE, VS

$B E, B E E$

$B E, B E E, B P E$

$B E, B P E, M E E$

BE, BEE, VS

BE, BEE

$B E, B E E$

$B E, B P E, V S$

BE, BEE

$B E, B E E, B P E, V S$

BE, BEE, BPE

$B E, B E E, B E P E, V S$

$B E, B E E, B P E, M E E, B E P E, V S$

$B E, B E E, B P E$

$\mathrm{BE}$

$B E, B P E$

$B E, B E E, B P E, V S$

BE: bosque de encino; BEE: bosque de enebro-encino; SBC: selva baja caducifolia; BPE: bosque de pino-encino; MEE: matorral espinoso-enebro; P: pastizal; BEPE: bosque de encino-pino-enebro; VS: vegetación secundaria; BEEME: bosque de enebroencino-matorral espinoso. * Nuevos registros para el estado de Hidalgo.

1Pueden considerarse como Pleopeltis pero aún no existe la transformación nomenclatural (Mickel \& Smith, 2004). 